\title{
Occurance of Mycotoxins and Mycotoxicosis in Poultry
}

\author{
Nikola Puvača ${ }^{1 *}$, Dragana Ljubojević ${ }^{2}$, Milica Živkov Baloš², Olivera Đuragić ${ }^{3}$, Vojislava Bursić ${ }^{4}$, Gorica Vuković ${ }^{5}$, \\ Radivoj Prodanović ${ }^{1}$ and Jelana Bošković ${ }^{1}$
}

${ }^{1}$ Department of Engineering Management in Biotechnology, University Business Academy, Serbia

${ }^{2}$ Scientific Veterinary Institute "Novi Sad", Serbia

${ }^{3}$ Scientific Institute for Food Technology in Novi Sad, Serbia

${ }^{4}$ Faculty of Agriculture, University of Novi Sad, Serbia

${ }^{5}$ Institute of Public Health of Belgrade, Serbia

Received: 畊 October 01, 2018; Published: 眥 October 09, 2018

*Corresponding author: Nikola Puvača, Faculty of Economics and Engineering Management, Department of Engineering Management in Biotechnology, University Business Academy, Novi Sad, Serbia

\section{Opinion}

Mycotoxins are biologically active, toxic metabolites produced by toxigenic fungi mainly belonging to Aspergillus, Fusarium and Penicillium species, which invade crops in the field and may grow on feedstuffs during storage under favourable conditions of temperature and humidity [1]. FAO estimated that about $25 \%$ of food and feedstuffs are contaminated with mycotoxins and strong efforts have been made to decontaminate them by the use of physical and chemical adsorbents but the success made so far is limited [2]. Like other environmental pollutants, mycotoxins also adversely affect the health and productivity in animals and esecially in poultry $[3,4]$. The economy of poultry industry is heavily affected due to wide mycotoxin exposure or contamination of various agricultural commodities. The economic losses are primarily due to the decreased growth rate, feed conversion efficacy, carcass yield, carcass quality and increased susceptibility to other diseases caused due to their immunosuppressive effects among the affected poultry. A mycotoxicosis is a disease caused by a natural toxin produced by a fungus. In poultry, this usually results when toxin producing fungi grow in grain and feed. Hundreds of mycotoxins have been identified, and many are pathogenic. Mycotoxins may have additive or synergistic effects with other natural toxins, infectious agents, and nutritional deficiencies. Many are chemically stable and maintain toxicity over time. Out of more than 350 mycotoxins identified in nature, aflatoxins, T-2 toxin, diacetoxyscirpenol, vomitoxin, zearalenone, ochratoxins, ergot alkaloids, oosporein, cyclopiazonic acid, and tricothecenes are the most common and important in poultry [5].

\section{Mycotoxicosis and Their Effect in Poultry}

Aflatoxicosis: The aflatoxins are toxic and carcinogenic metabolites such as Aspergillus flavus. Aflatoxicosis in poultry primarily affects the liver but can involve immunologic, digestive, and hematopoietic functions. Aflatoxin can adversely affect weight gain, feed intake, feed conversion ratio, pigmentation, carcass yield, egg production, male and female fertility, and large hatchability problems. Some effects are directly attributable to toxins, whereas others are indirect, such as reduced feed intake. Susceptibility to aflatoxins varies, but in general, ducklings, turkeys, and pheasants are susceptible, while chickens and Japanese quail are relatively resistant. Clinical signs vary from general unthriftiness to high morbidity and mortality. At necropsy the lesions are found mainly in the liver, which can be due to necrosis and congestion or yellow due to lipid accumulation. Hemorrhages may occur in liver and other tissues. In chronic aflatoxicosis, the liver becomes yellow to gray and atrophied.

Fusariotoxicosis: The genus Fusarium produces many mycotoxins injurious to poultry. The trichothecene mycotoxins produce caustic and radiomimetic patterns of disease exemplified by $\mathrm{T}-2$ toxin and diacetoxyscirpenol (DAS). Deoxynivalenol (vomitoxin, DON) and zearalenone are common trichothecene mycotoxins that are relatively nontoxic for poultry but may cause disease in large concetratin in feed. Fusariotoxicosis in poultry caused by the trichothecenes results in feed refusal, caustic injury of the oral mucosa and areas of the skin in contact with the mold, 
acute digestive disease, and injury to the bone marrow and immune system [3]. Lesions include necrosis and ulceration of the oral mucosa, gastro intestinal mucosa, mottling of the liver, atrophy of the spleen and other lymphoid organs, and visceral hemorrhages. In laying hens, decreased egg production can be accompanied by depression, recumbency, feed refusal, and cyanosis evident in the comb and wattles [6]. Other Fusarium mycotoxins cause defective growth of long bones.

Ochratoxicosis: Ochratoxins are quite toxic to poultry. These nephrotoxins are produced chiefly by Penicillium viridicatum and Aspergillus ochraceus in grains and feed. Ochratoxicosis causes primarily renal disease but also affects the liver, immune system, and bone marrow. Severe intoxication causes reduced spontaneous activity, huddling, hypothermia, diarrhea, rapid weight loss, and death. Moderate intoxication impairs weight gain, feed conversion ratio, pigmentation, carcass yield, egg production, fertility, and hatchability $[5,6]$.

Ergotism: Toxic ergot alkaloids are produced by Claviceps spp, which are fungi that attack cereal grains. The mycotoxins form in the sclerotium, a visible, hard, dark mass of mycelium that displaces the grain tissue. Within the sclerotium are the ergot alkaloids, which affect the nervous system, causing convulsive and sensory neurologic disorders, the vascular system, causing vasoconstriction and gangrene of the extremities and the endocrine system, including neuroendocrine control of the anterior pituitary gland. In chicks, the toes become discolored due to vasoconstriction and ischemia. In older poultry, vasoconstriction affects the comb, wattles, face, and eyelids, which become atrophied and disfigured. Vesicles and ulcers develop on the shanks of the legs and on the tops and sides of the toes. In laying hens, feed consumption and egg production are reduced [6].

\section{Mycotoxicosis Diagnosis in Poultry}

Mycotoxicosis should be suspected when the history, signs, and lesions are suggestive of feed intoxication, and especially when moldy ingredients or feed are evident. Toxin exposure associated with consumption of a new batch of feed may result in subclinical or transient disease. Chronic or intermittent exposure can occur in regions where grain and feed ingredients are of poor quality or when feed storage is substandard or prolonged. Impaired production can be a clue to a mycotoxin problem, as can improvement because of correction of feed management deficiencies. Definitive diagnosis involves detection and quantitation of the specific toxins. This can be difficult because of the rapid and high volume use of feed and ingredients in poultry operations. Diagnostic laboratories differ in their respective capabilities to test for mycotoxins and should be contacted before sending samples. Feed and also poultry that are showing sings of sicknes or recently dead should be submitted for pathological examinatios. A necropsy and related diagnostic tests should accompany feed analysis if mycotoxicosis is suspected
Concurrent diseases can adversely affect production and should be considered. Sometimes, a mycotoxicosis is suspected but not confirmed by feed analysis. In these situations, a complete laboratory evaluation can exclude other significant diseases. Feed and ingredient samples should be properly collected and promptly submitted for analysis. Mycotoxin formation can be localized in a batch of feed or grain. Multiple samples taken from different sites increase the likelihood of confirming a mycotoxin formation zone. Samples should be collected at sites of ingredient storage, feed manufacture and transport, feed bins, and feeders $[5,6]$.

\section{Prevention of Mycotoxicosis in Poultry}

Prevention of mycotoxicoses should focus on using feed and ingredients free of mycotoxins and on management practices that prevent mold growth and mycotoxin formation during feed transport and storage. Regular inspection of feed storage and feeding systems can identify flow problems, which allow residual feed and enhance fungal activity and mycotoxin formation. Mycotoxins can form in decayed, crusted feed in feeders, feed mills, and storage bins, cleaning and correcting the problem can have immediate benefits. Temperature extremes cause moisture condensation and migration in bins and promote mycotoxin formation. Ventilation of poultry houses to avoid high relative humidity also decreases the moisture available for fungal growth and toxin formation in the feed. Antifungal agents added to feeds to prevent fungal growth have no effect on toxin already formed but may be cost effective in conjunction with other feed management practices [6]. Propionic acid are effective inhibitor, but the effectiveness may be reduced by the particle size of feed ingredients and the buffering effect of certain ingredients. Sorbent compounds such as hydrated sodium calcium aluminosilicate effectively bind and prevent absorption of aflatoxin. Esterified glucomannan, derived from the cell wall of the yeast Saccharomyces cerevisiae, is protective against aflatoxin B1 and ochratoxins. It reduces toxicity through the binding and reduction in bioavailability of fumonisins, zearalenone, and T-2 toxin. Various other fermentation products, algae and plant extracts, and microbial feed additives have demonstrated ability to bind or degrade mycotoxins and may be applicable and appropriate for the situation.

\section{Acknowledgement}

The paper is a part of the research work on the project III 46012 financed by the Ministry of Education, Science and Technological Development of the Republic of Serbia.

\section{References}

1. Shamsudeen P, Shrivastava HP, Ram S, Chandra D (2013) Effect of chelated and inorganic trace minerals on aflatoxin synthesis in maize. J Poult Sci Tech 1: 13-16.

2. Shetty P, Jespersen L (2006) Saccharomyces cerevisiae and lactic acid bacteria as potential mycotoxin decontaminating agents. Trends Food Sci Technol 17: 48-55. 
3. Zain ME (2011) Impact of mycotoxins on humans and animals. J Saudi Chem Soc 15: 129-144.

4. Katole SB, Kumar P, Patil RD (2013) Environmental pollutants and livestock health: a review. Vet Res I 1: 1-13.

5. Ljubojević D, Jakšić S, Živkov Baloš M, Mihaljev Ž, Puvača N, et al. (2014) Presence of aflatoxins, zearalenone, ochratoxin A and trichothecenes in corn (Zea mays) in Republic of Serbia. International Symposium "Feed Technology", Novi Sad, Serbia 16: 193-197.

6. Puvača N, Nikolova N, Kostadinović Lj, Đuragić O, Popović S, et al. (2016) Productive performance of laying hens fed by dietary mycotoxins binder MIN-A-ZEL: Internatinal Agricultural Symposium, AgroSyim2016, Jahorina, Bosnia and Herzegovina 7: 1014

\section{(c) (i) \\ This work is licensed under Creative Commons Attribution 4.0 License \\ Submit Article}

Submission Link:

DOI: $10.32474 /$ CDVS.2018.02.000130

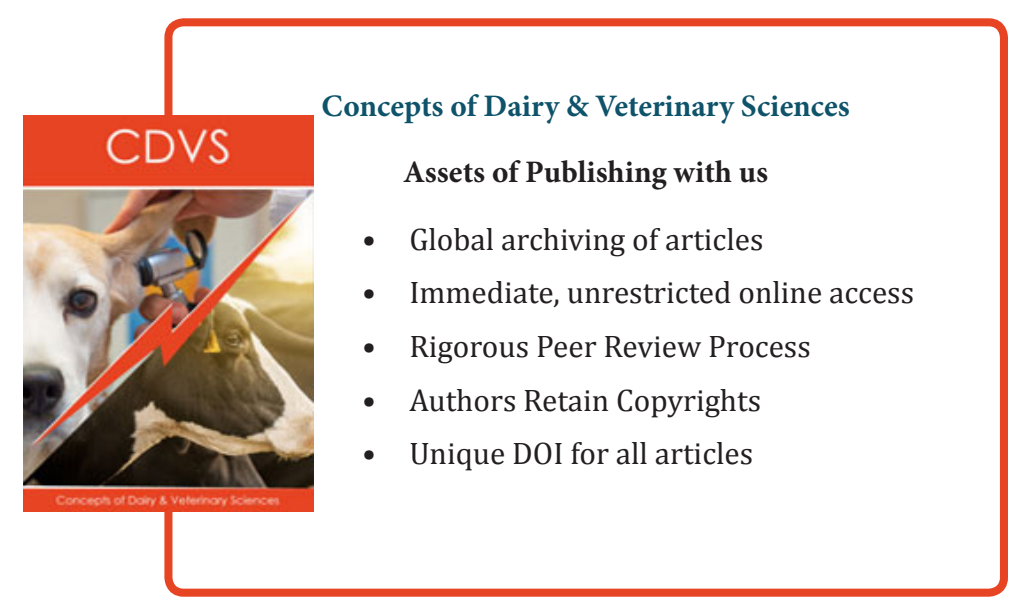

